\title{
Upgraded Force on a Wall from Reflected Surface Gravity Waves
}

\author{
Kern E. Kenyon \\ 4632 North Lane, Del Mar, CA, USA
}

Correspondence to: Kern E. Kenyon, kernken@aol.com

Keywords: Reflected Wave Force, Surface Gravity Waves

Received: December 19, 2018 Accepted: January 18, 2019

Published: January 21, 2019

Copyright $\odot 2019$ by authors and Scientific Research Publishing Inc.

This work is licensed under the Creative Commons Attribution International License (CC BY 4.0).

http://creativecommons.org/licenses/by/4.0/

\section{(c) (i) Open Access}

\section{ABSTRACT}

During reflection from a solid vertical wall, surface gravity waves produce a steady force on the wall, which was calculated earlier by the momentum method. Recently the linear momentum of these waves has been estimated to be twice as large as deduced from the classical Stokes drift formula. Therefore, the magnitude of the reflected wave force has been doubled here and the algebraic form of the equation is converted to be more understandable physically. Wave force equals fluid density times the square of the particle's orbital speed and a numerical constant. Observations are welcomed to compare with the theory.

\section{INTRODUCTION}

There would seem to be a certain amount of urgency in better understanding the magnitude of the force on a wall caused by the reflection of surface gravity waves hitting the wall. One reason for urgency is the potential practical application of the force equation to various marine structures on beaches and in harbors within the field of ocean engineering. Another reason is to clear up the conceptual confusion springing from the existence of so many available theories of the wave force. Before 2004 there were published more than 25 different formulas for the wave force all based on the Eulerian or Lagrangian forms of the fluid dynamic equations [1]. Then one more theory was added to the literature by me, but it was founded on an entirely different method: the momentum balance [2]. Since then no information has come to my attention that leads to a clarification of this awkward and embarrassing scientific situation.

Any time now the burden of future work on the wave force dilemma logically shifts to the shoulders of the experimentalists. Meanwhile a recent unexpected theoretical result [3] can be used to modify the earlier force formula derived from the momentum balance, because it has been found that the linear momentum of surface gravity waves is very likely twice the value obtained from the Stokes drift equation that was derived over 170 years ago.

\section{MOMENTUM BALANCE}

If a time rate of change of momentum occurs, it takes a force to cause this. Not only do surface gravity 
waves possess linear momentum, but they also transport it in the direction of wave propagation. At a wall during wave reflection the sign of the momentum reverses because the direction of propagation reverses. Since the wall causes the reflection by applying a force to the wave, action equals reaction (Newton's $3^{\text {rd }}$ law), dictates that there is an equal but opposite force from the wave back on the wall.

What exactly is this linear momentum of a surface gravity wave? It started with Stokes (1847) [4]. In a mathematical examination of propagating surface gravity waves he made a calculation that the orbits of the fluid particles are not completely closed circles, in such a way that at the end of a wave period there is a tiny advancement of fluid in the direction of wave travel, which since then has been called the Stokes drift. Much later the reason why the Stokes drift occurs was explained physically [5] independent of the original mathematics.

All the characteristics of a propagating surface gravity wave, including the size of the particle orbits, have been observed, over a period of several centuries, to decrease with increasing depth down form the air/water interface, and to essentially vanish below a depth equaling approximately one wavelength. Consequently, at a wave trough, which is one orbital diameter lower than a crest, the backward speed is a little smaller than the forward speed is at the crest, resulting in a net steady shift of mass in the forward direction (in the reference frame fixed to the ocean bottom). Visualization of the forward shift is easily carried out in a wave tank with the help of small neutrally buoyant particles.

When the derivation of the wave force on a wall was carried out using the momentum method [2], the classical Stokes drift was incorporated into the time rate of change of the linear momentum. Then recently a new estimate of the "Stokes" drift [3], from a different model than used by Stokes, predicted a value of the drift to be nearly twice as big as Stokes found it to be. Therefore, the purpose of the present note is to upgrade the wave force equation by a factor of two, because knowing about this may be of practical value in the future, depending on how the confirmation between theory and observations turns out. Also the equation for the wave force is converted into a form that is probably more understandable from a physics perspective.

\section{UPGRADED WAVE FORCE}

At the mean free surface the new estimate of the "Stokes" drift $U$ is [3]

$$
U=2(a k)^{2} V
$$

where $a$ is the wave amplitude and $k$ is the wave number. In the reference frame where the wave shape is fixed, with fluid underneath flowing past the observer, $V$ is the forward speed at the crest. Equation (1) differs from the classical one by the factor of 2 and the phase speed $c$ replaces $V$. For small mean wave slopes, $(a k), V$ is smaller than $c$ but not by much. Therefore, the main difference is the factor of 2 in (1). Notice that (1) is independent of the horizontal coordinate as well as being independent of the time.

Multiply (1) by the constant fluid density $\rho$ to get the linear momentum, $m$. Then twice the momentum divided by the wave period $T$ gives the time rate of change of the momentum at the wall over a wave period.

$$
\frac{\mathrm{d} m}{\mathrm{~d} t}=\frac{1}{T} 4 \rho(a k)^{2} V
$$

Finally, integrating (2) over the horizontal distance of one wavelength $L$ adjacent to the wall produces the force per unit vertical area $f$ by the reflected wave on the wall at the mean free surface

$$
f=4 \rho(a k)^{2} c^{2}=4 \rho(a \omega)^{2}
$$

where $c=L / T=\omega / k$ and the approximation $V \approx c$ has been made. Equation (3) is a factor two times the earlier result [2] due directly to the fact that the linear momentum used is now twice as large. Assuming the amplitude and frequency of the wave are constants, then Equation (3) depicts the parameters of a steady force. 
Another way to write (3) incorporates the realization that $a \omega=u$ is the orbital speed of a fluid particle at the surface, because $a \omega=2 \pi a / T$. Therefore

$$
f=4 \rho u^{2}
$$

Which is a bit shorter than (3) and perhaps physically more attractive as well. In fact in appearance (4) is very similar to the well-known empirical formula for the wind or water force $F$ on a solid object

$$
F=C_{D} \rho U^{2}
$$

where $U$ is the mean flow speed, $\rho$ is the fluid density and $C_{D}$ is a numerical constant called the drag coefficient. Also the lift force on a circular arc wing has been put into the same format as (5), except that the drag coefficient is replaced by a different numerical constant [6].

In addition the upgraded Stokes drift (1) can be written

$$
U \approx 2(a k) u
$$

Showing that the drift is small compared to the particle speed due to the smallness of the mean wave slope $a k$ and much smaller compared to the phase speed [see Equation (1)] which is proportional to (ak) ${ }^{2}$.

\section{CONCLUSION}

A previous formula for the reflected wave force on a wall, calculated by the conservation of momentum method applied to propagating surface gravity waves, is increased in magnitude by a factor of two due to a recent re-evaluation of the Stokes drift, which is directly related to the linear momentum of these waves. Also the wave force equation is converted into a physically more understandable format: force equals the fluid density times the square of the orbital particle speed and a numerical constant. Although this functional form is unique to the wave reflection problem, there are similarities in other cases such as the well-known force of fluid flow past solids involving the drag coefficient, the density and the square of the mean flow speed. Much needed now are wave measurements, presumably in the laboratory, to hold up to the wave reflection theory.

\section{ACKNOWLEDGEMENTS}

Comments by the Reviewer led to significant changes which improved the text.

\section{CONFLICTS OF INTEREST}

The author declares no conflicts of interest regarding the publication of this paper.

\section{REFERENCES}

1. Rundgren, L. (1958) Transactions of the Royal Institute, No. 122 (Stockholm, Sweden).

2. Kenyon, K.E. (2004) Force and Torque on a Wall from Reflected Surface Gravity Waves. Physics Essays, 17, 95-102. https://doi.org/10.4006/1.3025646

3. Kenyon, K.E. (2018) Stokes Drift: A New Estimate. Natural Science, 10, 452-454. https://doi.org/10.4236/ns.2018.1012045

4. Stokes, G.G. (1847) On the Theory of Oscillatory Waves. Transactions of the Cambridge Philosophical Society, 8, 441-455.

5. Barnett, T.P. and Kenyon, K.E. (1975) Recent Advances in the Study of Wind Waves. Reports on Progress in Physics, 38, 667-729. https://doi.org/10.1088/0034-4885/38/6/001

6. Kenyon, K.E. (2017) Lift Force on a Circular Arc Wing. Natural Science, 9, 351-354. https://doi.org/10.4236/ns.2017.910033 